\title{
Sign Language Phonetic Annotation meets Phonological CorpusTools: Towards a Sign Language Toolset for Phonetic Notation and Phonological Analysis
}

\author{
Oksana Tkachman, ${ }^{1}$ Kathleen Currie Hall, ${ }^{1}$ André Xavier, ${ }^{2}$ \\ and Bryan Gick ${ }^{1,3}$ \\ ${ }^{1}$ University of British Columbia, ${ }^{2}$ University of Sao Paulo, ${ }^{3}$ Haskins Labs
}

\section{Introduction}

The field of sign language linguistics is still very young, and many tools and methods that have been available to spoken language linguists for decades are yet to be adapted or developed for their signlanguage colleagues. One such tool that is lacking is a unified system of phonetic transcription of the formational features of signs. There is a growing understanding in the field that some kind of a common notation system is required in order to enable cross-project and cross-linguistic comparison of data. Without such a common notation system there is no way to ensure that the results of individual projects are adequately reported, and that cross-linguistic and universal claims and models are made on comparable datasets. The lack of a unified and universally accepted system, analogous to the IPA for spoken languages, results in much research that focuses on theoretical issues of sign language linguistics rather than physical output mechanisms (Tyrone 2002), and it has especially heavy consequences for the fields of sign language phonetics and phonology. Existing studies often use notation systems that are only comprehensible to the researchers using it, and they report their results in very general ways with only a few line pictures, captions, or even verbal descriptions of the signs to illustrate some specific points (Garcia \& Sallandre 2012). The reader might get a general understanding of the findings, but the details are obscure and replications and comparisons are difficult if not impossible.

Another problem that results from the absence of a sign language IPA is felt in corpus studies and experimental approaches. As technology becomes more accessible to researchers and more kinds of software are developed for linguistic research, the need for adequate notation of the data becomes especially pronounced. However, new technology often brings more questions than answers. At the moment, several big sign-language corpora are being assembled in different countries and for different sign languages (e.g., for Australian Sign Language (Auslan)). ${ }^{1}$ The focus of those corpora, however, is mostly syntax, semantics, and discourse, which is a natural outcome of the fact that those corpora often aim to answer more general questions of sign language structure, and to help with standardization and recognition of sign languages. This approach to corpus construction, however, often results in modes of annotation that are the opposite of what sign language phoneticians and phonologists would wish for. One such notable practice is lemmatization, the practice of assembling all related phonetic, phonological and morphological variants under one lemma (Johnston 2008), thus losing all the information on different variants of the same sign, the environment in which the sign was produced, etc. Beside the issue of lemmatization, sign language corpus studies face issues of optimization of data annotation tasks, automated processing of large corpora, and the possibility of sharing corpora for cross-linguistic comparisons (see Garcia \& Sallandre 2012 for a detailed elaboration on these three issues, and Frishberg, Hoiting, \& Slobin 2008). Thus, the field is under pressure not just to develop a reliable notation system, but also to make this notation system suitable for mechanization of data storing and processing (see also Hochgesang 2014).

Another interesting development in the field is the use of technology such as dataglove (Eccarius, Bour, \& Scheidt 2012) or various motion-tracking systems; and analyzing such data provides new insights

\footnotetext{
${ }^{1}$ http://www.auslan.org.au/about/corpus/

(C) 2016 Oksana Tkachman, Kathleen Currie Hall, André Xavier, and Bryan Gick

Proceedings of $A M P 2016$

Completed March 31, 2016
} 
for sign language linguists. However, these technologies are mostly restricted to controlled, artificial data, which can have undesirable consequences for phonological and phonetic analyses. Additionally, these new technologies are expensive and not available to everyone, especially in fieldwork situations. The most common way of gathering data in the field is still simple videotaping signers in natural, controlled and semi-controlled environments. Coming up with a system applicable for such videotaped data will enable the field to approach the data already gathered and stored with new questions and new approaches, as well as to analyze videos of signers available through public domains such as the YouTube. Despite all the technological advancements, the need for a more traditional, cheap, and easily available notation system such as IPA is still pertinent.

What we argue for in this paper is a common notation system for formational features of signs, that is easily adaptable for linguistic software and enables researchers both to create large corpora of annotated data and to compare those corpora across different projects both within a single sign language and across different sign languages (see also Hochgesang 2014, Eccarius \& Brentari 2008, Johnson \& Liddell 2010 for a similar argument). We also argue that adopting such a system will make researchers more mindful of the ways they treat and report data. We report our suggested adaptation of Sign Language Phonetic Annotation (Johnson and Liddell 2011, 2012) for Phonological CorpusTools (Hall et al. 2015) as a possible means for achieving those goals.

\section{Background}

The need to transcribe signs in some written form has been recognized from the very inception of the field of sign language linguistics, with the pioneer of the field, William Stokoe, coming up with the first such attempt, usually referred to as 'the Stokoe system' (Hochgesang 2014). In later years, a few additional attempts to create an adequate notation system for sign languages were undertaken, such as the Hamburg Notation System (HamNoSys, Prillwitz et al. 1989), the Prosodic-Model based transcription (Eccarius and Brentari 2008), and the system based on the Movement-Hold Model (Liddell and Johnson 1989). All these systems are still in use by some researchers, but none has been universally adopted, mostly because none is entirely adequate for phonetic data, and especially for cross-linguistic comparisons (see Hochgesang 2014 for a detailed evaluation of these systems). As a result, most sign language studies dealing with phonetic data rely on phonemic notations usually based on the manual alphabet of the surrounding spoken language, a situation which is both inadequate representationally and inaccurate in that it imposes phonological assumptions on phonetic data. It also makes cross-linguistic comparisons difficult, especially comparisons of handshapes that do not look exactly like a specific manual letter but are described as a variation of such a letter.

One of the recent attempts to create a notation system for signs, developed by Johnson and Liddell (2011a, 2011b, 2011c, 2012) and called here Sign Language Phonetic Annotation, or SLPA (following Hochgesang 2014), seems more successful than others in its pursuit of a pure phonetic notation. In the first article of the series, the authors stated their intention to offer a complete phonetic system; however, as of now only the handshape coding is available. Signs have four phonological categories, or parameters: handshape, location, movement (Stokoe 1960), and orientation (Battison 1978). Changing any one of these can change the meaning of the sign, and minimal pairs are found for each of those parameters in most sign languages (Sandler and Lillo-Martin 2004). Handshape is probably the most prominent phonological category of the sign, and it has special properties in sign language processing: handshape facilitates sign recognition in lexical decision tasks and priming studies (Carreiras et al. 2008) and picture-naming inference tasks (Baus et al. 2008). Handshape is also last to be acquired in first language acquisition and first to be lost in language disorders: handshape is acquired later and least accurately than other phonological categories such as location by deaf children acquiring sign languages natively (Marentette \& Mayberry 2000, Meier 2000); it is the most sensitive of all phonological parameters to age of acquisition (Brentari 2006); and handshape errors are the most common errors in aphasia (Corina 2000). Therefore, in an annotation system restricted to only one phonological category handshape appears to be the natural choice to start with (see also Brentari and Eccarius 2010).

SLPA is much more descriptive and therefore phonetic than other systems that build on numerous theoretical assumptions and are more dependent on abstract representation of the data (Johnson \& Liddell 2011b, Hochgesang 2014). SLPA focuses on several subcomponents of the handshape, or hand 
configuration (HC): (1) the configuration of each of the four fingers, based on extension and flexion of each visible joint and on abduction/adduction between those fingers; (2) the presence of thumb-finger contact and the manner of this contact; (3) the configuration of the thumb (akin to fingers, based on extension and flexion of the visible joints and adduction, abduction or opposition of the thumb to the palm); and (4) the hand configuration extension (see Figure 1).

\section{[HC extension] [thumb configuration] [thumb/finger contact] [configuration of fingers]}

Figure 1. Schematic subcomponents of the phonetic notation of HCs in the notational order, as proposed in Johnson and Liddell (2012)

As an example, consider the hand configuration shown in Figure 2, which shows the letter $\mathrm{T}$ in Brazilian Sign Language. This would be transcribed as [U<fh bd $\varnothing /$ um1 1FEE<2EEE/3EEE/4EEE] in SLPA (Johnson \& Liddell 2012). The reader is directed to the Johnson \& Liddell papers for a complete description of the symbols used here, but as a brief example, the sequence [ $44 \mathrm{EEE}]$ in the above transcription indicates that the fourth finger (the "pinkie" finger, symbolized [4]) has all three of its joints fully extended ([E]), and that it is widely abducted away from the third finger $([\langle])$. This system is therefore somewhat analogous to a hypothetical system for spoken languages that would include information about the voicing, place of articulation, and manner of articulation of consonants as separable pieces of a single transcription-e.g., encoding a [t] as an Unvoiced-Alveolar-Stop, or [UAS], rather than using a single arbitrary character to represent all of that information simultaneously.

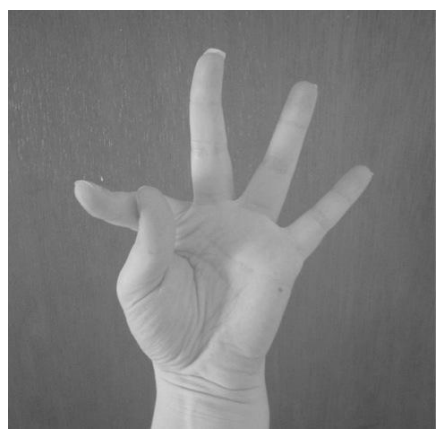

Figure 2: The letter $\mathrm{T}$ in Brazilian Sign Language.

Johnson and Liddell (2011b: 41) claim that using SLPA they were able to notate every handshape they came across while developing the system. The system is indeed so detailed that it is possible to both notate any handshape and reconstruct any handshape from its notation. It is also intuitive and easily learned (Hochgesang 2014), and the use of the system does not require the coder to know any sign language, which is not the case for many other coding systems (see Frishberg, Hoiting, and Slobin 2012). Additionally, unlike other systems, SLPA uses a small number of symbols for notation, and (almost) all of them are found on a standard keyboard. However, the great detail of SLPA is both its strength and its weakness, the issue that is discussed below.

\section{Shortcomings of SLPA}

Ironically, the very features that make SLPA so very successful in capturing phonetic detail are also features that undermine its effectiveness when used by many coders in notating significant amounts of data. Johnson and Liddell argue that even though only linguistically relevant information should be included in a notation system, it is probably necessary to start with more information and reduce the description as certain phenomena are found not to be linguistically relevant. As a result, their system is too exhaustive, requiring between 23 and 34 symbols for each possible handshape, and capturing handshapes that are implausible in terms of being linguistically meaningful, either because they are perceptually nondistinctive in spite of being anatomically different or because they are anatomically impossible to produce. 
Consequently, while allowing for an extraordinary amount of phonetic detail, it is hard to capture patterns and make generalizations with this notation system, even basic ones such as allophonic variants of the same phoneme.

Our objective, therefore, is to make SLPA more linguistically relevant without losing valuable phonetic detail. Below we discuss some issues arising from using SLPA, along with our suggestions on how to deal or adapt the system to be more linguistically relevant, and how we intend to implement those suggestions in Phonological CorpusTools (PCT) in our future work.

3.1 Too many degrees of freedom SLPA is the least compact notation system of the ones frequently used by sign language researchers (Hochgesang 2014). Each handshape requires between 23 and 34 symbols in SLPA to be adequately annotated. Having 34 symbols used for notating a single handshape means there are 34 possible locations for errors for that handshape. While the system is a bit cumbersome because of its length, it is relatively easy to learn from the perspective of understanding the meaning of each symbol (see also Hochgesang 2014 for the same observation), but mistakes are easy to make, especially when a coder is notating a large dataset. The long character strings are also difficult for humans to read, which also calls for some sort of machine processing. SLPA in its original form also cannot produce a broad (phonemic) notation (Hochgesang 2014), which can have consequences for the types of analysis that can be done on the data annotated with SLPA.

3.2 Anatomically impossible handshapes The great freedom of SLPA allows the coder to create annotations for handshapes that are anatomically impossible. These configurations are analogous to the shaded boxes in the IPA chart for spoken languages, but unlike such sounds, they are perfectly possible to transcribe in SLPA. Moreover, since each finger and each joint is annotated separately from each other, it creates a false impression that each finger and joint is capable of the same degrees of inflections and flexions, an impression that less anatomically savvy users have no reason to doubt, which can lead to even more mistakes than simple mistranscriptions of the kind we mentioned earlier.

SLPA does not take into account the fact that fingers are not the same anatomically, not just in terms of length and size, but also in terms of freedom of movement. One of the reasons for this shortcoming of the SLPA is that the authors based the system on joints and did not take into account the muscles of the hand. However, the muscle structure determines much of what human hands are capable or incapable of doing. For example, Ann (2000) explains patterns in sign language handshapes in terms of hand muscle structure. There are separate extensor and tendon muscles for the index finger and the little finger, which allow them to extend independently; but in order to extend either the middle finger or the ring finger, an extensor muscle that is shared by all fingers has to be applied, while other muscles simultaneously flex the rest of the fingers. This means that when either the middle finger or the ring finger is fully extended, the rest of the fingers cannot be fully flexed (see Figure 3). (To see this for themselves, the reader is invited to extend their middle or ring finger while trying to fully flex the rest of the fingers, and to compare the ease of doing so with the ease of extending the index finger and fully flexing the other fingers. In most people, fully flexing the rest of the fingers will force the ring finger to partially flex as well.)

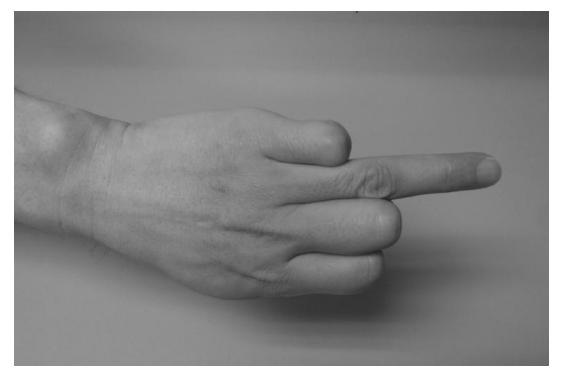

Figure 3. An extended ring finger. As can be seen in the picture, the rest of the fingers cannot be fully flexed while the ring finger is fully extended. The same is true for the middle finger.

Anatomically impossible combinations are hard to control for if the coder does not know such details 
and if there is no superimposed control over what is permissible in the annotation. Making such control automatic will improve quality of notation without making all the users of the system proficient in human hand anatomy. We will discuss some possible solutions below.

3.3 Redundant forms Redundant notations are also related to the issue of hand anatomy. For example, in order to flex the distal joints, the medial joints usually have to be flexed first; therefore specifying flexion of medial joints when the distal joints are flexed is redundant. A counter-example of a distal joint being flexed with a medial joint extended can be seen on Figure 4; this is not entirely anatomically impossible, but people who do not have a torn tendon or a flexion deformity such as a Maller finger are unlikely to be able to do it. Though of interest for research on atypical populations, such handshapes are unlikely to be linguistically relevant if most of the normal population is unable to even produce them.

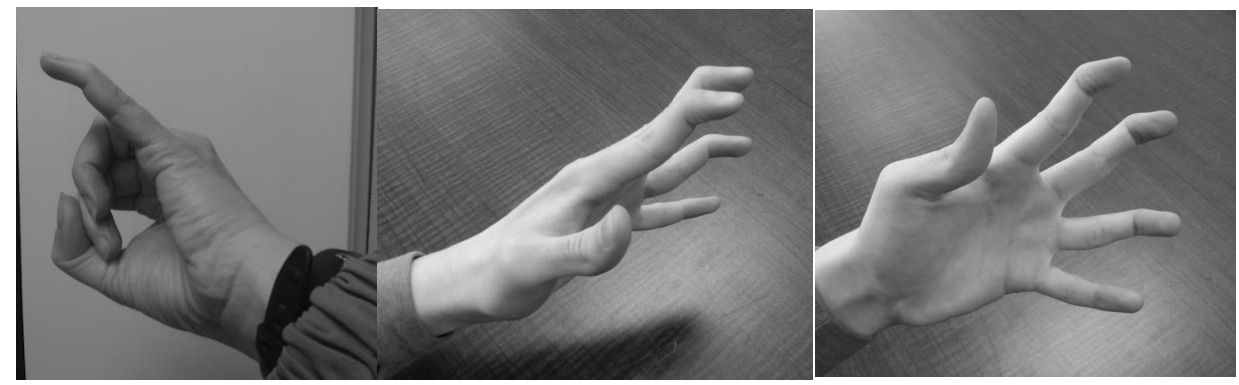

Figure 4. Mallet finger (also known as baseball finger). The distal joint is flexed while the medial joint is extended.

3.4 Perceptually nondistinctive forms A fourth problem is that of forms that are perceptually nondistinctive, an issue that arises from the set-up of the SLPA system itself. SLPA was created to capture as much phonetic detail as possible; for instance, Johnson and Liddell followed Eaton (2007) in incorporating different degrees of joint flexion into the coding system. Each joint of each finger is specified as one of six possible degrees of flexion: fully hyperextended $(\mathrm{H})$, partially hyperextended $(\mathrm{h})$, fully extended $(\mathrm{E})$, partially extended (e), partially flexed (f), or fully flexed (F). ${ }^{2}$ For proximal joints (that is, joints closest to the palm), it is relatively easy to distinguish multiple degrees of flexion, because the difference between any two adjacent degrees of flexion is approximately $30^{\circ}$ of motion (Johnson \& Liddell 2011b: 28). For distal joints, however, these degrees are harder to differentiate, with the difference between any two adjacent degrees of flexion being as small as $10^{\circ}$ of motion.

Making such a decision for distal joints is difficult even for a still picture of high quality with the joint in question in full view, but it is virtually impossible if the quality of the data is not so high, especially as the distal joints are the smallest. Distinguishing forms such as partially extended and partially flexed, especially for distal joints, in the notation system is likely to obscure linguistic analyses rather than elucidate them, as different transcribers will be more likely to be inconsistent with each other, even if they manage to be internally consistent, leading to incomparable transcriptions.

In addition to the difficulty coding such differences, there is also a question of their perceptual salience linguistically. As signers tend to look each other in the face during a signing conversation, the handshapes that are below the face area are generally perceived with peripheral vision, which is not very sensitive to fine details, and therefore many handshapes that are anatomically different are not perceived as distinct from each other (Siple 1978). Determining which types of handshape differences are perceptually nondistinctive will help to reduce the number of unnecessary phonetic details and make finding phonological patterns easier.

\footnotetext{
${ }^{2}$ Those degrees were proposed to meet the assumption that these features are binary.
} 


\begin{tabular}{|l|l|l|}
\hline Symbol & Proximal joints & Distal joints \\
\hline E & $0^{\circ}$ & $0^{\circ}$ \\
\hline e & $+30^{\circ}$ & $+10^{\circ}$ \\
\hline f & $+60^{\circ}$ & $+20^{\circ}$ \\
\hline F & $+90^{\circ}$ to $100^{\circ}$ & $+45^{\circ}$ to $80^{\circ}$ \\
\hline
\end{tabular}

Table 1. Approximate midpoints for the extension and flexion of the proximal and distal joints (adopted from Johnson \& Liddell 2011b).

Finally, retaining all six degrees of flexion conflicts with the aim of making the notation as theoryneutral as possible, a problem that can keep the system from use by researchers who do not subscribe to a given theory or claim. Johnson and Liddell themselves criticize notations based on theoretical assumptions such as selected fingers for describing signs (see Johnson and Liddell 2010 for the full criticism). The focus on joints and flexions makes SLPA one of the most theory-neutral systems proposed, yet the six degrees of flexion are based on the assumption that phonetic features are binary. The authors introduce three features of finger configuration, those of flexion, hyperextension and muscular limit: flexion and hyperextension refer to whether a particular joint is flexed or extended, and muscular limit refers to the extent to which the joint is flexed or extended. For example, if the joint is flexed to its full muscular limit (that is, it is anatomically impossible to flex it any further), it is coded as F, but if its muscular limit is not reached, it is coded as $\mathrm{f}$ (see Table 2). In this case the three configurational features are taken to be [+flexed, hyperextended, +muscular limit] for F and [+flexed, -hyperextended, -muscular limit] for f. However, treating flexion of a joint with at least $100^{\circ}$ of total range of motion as a binary feature is ignoring the gradient nature of movement. For instance, the authors treat e-flexion (that is, partially extended flexion) as different from f-flexion (that is, partially flexed flexion) because e-flexion is [-flexed]. However, it is not really [-flexed] because the joint is not fully extended (as E-flexion is) (see Table 1).

\begin{tabular}{|l|l|}
\hline Features & Configurations \\
\hline$[+$ flexed $]$ & f/ F \\
\hline$[$-flexed $]$ & e / E \\
\hline$[+$ hyperextended $]$ & h / H \\
\hline$[+$ muscular limit $]$ & F, E, H \\
\hline
\end{tabular}

Table 2. The coding of binary features in SLPA (adopted from Johnson \& Liddell 2011b).

The notion of muscular limit is not very helpful either. An extended joint is not in fact at its muscular limit, since it is possible to push it even further to create a hyperextended configuration. Similarly, hextension and $\mathrm{H}$-extension (the two degrees of hyperextension) are supposed to be different realizations of the muscular limit binary feature. However, both of them are at the muscular limit, and it is not clear how h-extension can be claimed to be [-muscular limit] if E-extension, which requires less effort to produce, is defined as [+muscular limit]. Given these issues, it is preferable to drop the entire binary assumption behind the six degrees of flexion altogether. Without the binary assumption, there is also no need for all six of degrees. We believe that four degrees of flexion (hyperextended, extended, flexed, and intermediate between flexed and extended) will suffice for the joint description and help to avoid bringing theoretical assumptions into the notation system (see section 4.1).

The proposed changes are along the lines of the systems' authors opinion that their description should eventually be reduced to account only for the details that are linguistically relevant (Johnson \& Liddell 2010). Our suggestions, therefore, are the first step towards this goal of making SLPA more functional. 


\section{Proposed modifications to SLPA: general suggestions}

In this section we outline some general suggestions for modifying SLPA. Some of them can be applied in any version of SLPA, even in manual coding (section 4.1), whereas others are PCT-specific, though they can also be applicable in any software adaptation of SLPA (section 4.2).

4.1 Merging intermediate degrees of flexion As has been argued above, we propose to drop the binary assumption behind the degrees of flexion. This change will be both more in line with Johnson and Liddell's quest for a theory-neutral annotation system as well as being a more anatomically realistic and easy to code implementation. Instead of the six degrees of flexion of the original SLPA (F, f, e, E, h, H), we propose to reduce their number to only four: $\mathrm{F}$ for fully flexed joints, $\mathrm{E}$ for fully extended joints, $\mathrm{H}$ for hyperextended joints (thus merging $\mathrm{h}$ and $\mathrm{H}$ configurations into one), and $\mathrm{m}$ for medially flexed joints (thus merging e and f configurations into one). We argue for those changes on the basis of the following reasons.

As mentioned in section 3.4, such modifications can decrease the number of perceptually nondistinctive handshape configurations captured as distinctive in the current version of SLPA. The differences between partially extended and partially flexed joints, as well as partially and fully hyperextended joints, are hard to see even on still pictures, let alone during a natural signed communication, and are virtually impossible to see in distal joints which are both smaller in size and more limited in degree of movement. Keeping those distinctions slows down human coders who have to make nonintuitive decisions about the appropriate symbol for ambiguous flexions. This in turn encourages variability in coding, so that the same handshape coded by different people can appear in different annotations and can be treated as two distinct handshapes in further analyses. Reducing the number of symbols used will also make the system more human-readable, which at present is one of the greatest weaknesses of SLPA (see Hochgesang 2014).

Eliminating those distinctions in annotation will simplify the system, make it easier both to use and to read, and will not result in any meaningful loss of phonetic information.

4.2 Controlling for anatomically impossible handshapes The question of which handshapes are anatomically possible and which are not is not widely discussed in the field at the moment. Yet, having a better understanding of the anatomical allowances and constraints would be very beneficial for research on sign language phonetics and phonology. Having a list of possible and impossible handshapes, as least in the framework of the annotation system, would be the first such step towards filling this gap in our knowledge. This issue is especially critical for an annotation system such as SLPA, where each of its numerous symbols is inserted independently, opening up many possibilities for errors and misspellings. Creating an exhaustive lists of impossible handshapes can be seen as a daunting task, but we can at least start with simple generalizations. For example, a proximal joint can be hyperextended while the rest of the finger's joints are flexed, but a distal joint cannot be hyperextended while the rest of the joints are flexed (at least not without contact with the palm or a finger; the reader is invited to test this observation). In terms of SLPA, this means that combinations such as [1HFF] are possible but combinations such as [1FFH] (without contact) are not. Having a lists of such generalizations can help coders avoid at least some errors, especially in the early stages of familiarizing with the system. Of course, controlling errors with such lists would be even more doable with software (see below).

\section{Proposed modifications to SLPA: PCT-specific suggestions}

The Phonological CorpusTools software (PCT; Hall et al. 2015) allows researchers to make fast, replicable analyses of various phonological patterns, such as the predictability of distribution and functional load of phonological units (e.g., for quantifying degrees of contrastivity vs. allophony in a language) and the similarity of phonetic or phonological strings (e.g., for use in calculating neighbourhood density). By creating a relatively fine-grained, consistently applicable, and, importantly, unicode-character-based transcription system, these same measurements can be applied to sign language corpora. This will allow both for the documentation and analysis of individual languages and also for the comparative analysis of different languages, allowing greater understanding of the physiological vs. phonological patterns in sign language handshape. 
We believe that adopting SLPA to PCT in a way that takes the issues discussed above into account will make phonological analysis of handshape easier in general, but will also have the benefit of making computational approaches to such analysis feasible. Here we elaborate in more detail on this proposed adaptation.

5.1 Reducing degrees of freedom (Preset templates to choose from) In our proposed adaptation of SLPA to PCT, we would provide the user with pre-set templates to choose from, rather then forcing the user to manually enter up to 34 symbols one by one. This arrangement both eases notation process and helps reduce the amount of errors. The proposed implementation will also reduce the variability within the system itself. As has been mentioned above, the current version of SLPA requires anywhere between 23 and 34 symbols for a single handshape, which creates considerable differences in length for individual signs. In our implementation of SLPA in PCT, we will have the same length of annotation for all handshapes, 34 symbols. By requiring transcriptions to be in this more templatic format, we can ensure unambiguous machine readability. For example, an $[E]$ in slot 18 will always mean that the proximal interphalangeal (PIP) joint of the first (index) finger is fully extended-whereas without a templatic system, slot 18 could be the slot for any of a number of joints, and the PIP joint specification would be identified only as the second slot after the rightmost occurrence of the digit 1 . This would not be impossible to implement, but the templatic version makes computation easier.

The templates will be not for 34 symbols individually; instead, seven slots will be provided, each slot representing a meaningful segment of a handshape, in the order of the original SLPA system: arm, thumb configuration, thumb-finger contact, and each of the four fingers (see Figure 5).

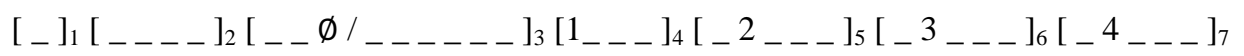

Figure 5. The proposed template for SLPA handshape annotation in PCT. The slots are, in order: (1) arm, (2) thumb configuration, (3) thumb-finger contact, (4) index finger, (5) middle finger, (6) ring finger, (7) little finger.

5.2 Controlling for erroneous forms Limiting human coders to a pre-set list of selectable templates to choose from will also allow effective control for erroneous forms. If the coder does not have an option of an anatomically impossible form for an individual slot, or an impossible combination of possible slots, s/he will have fewer chances to make errors. Of course, there is still a possibility to choose an erroneous possible form, but such human error is nearly impossible to control for. One of the ways to control for such situations is to provide a visual representation for each template. In this way, the coder would be able to compare the target handshape with the representation chosen and ensure that the representation was chosen appropriately. Such visual aids will be especially important in early stages of coders' training and for cases of between-coder disagreements. For example, one could choose the [1EEE] code, accompanied by a graphic such as that in (1a), to automatically set all of the joints of the index finger to full extension, or select the $[1 \mathrm{EEE}][=2 \mathrm{EEE}][=3 \mathrm{EEE}][=4 \mathrm{EEE}]$ code, accompanied by a graphic such as that in $(1 \mathrm{~b})$, to automatically set all of the joints of all four fingers to full extension and adduction.

(1) a.

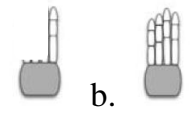

\section{Conclusion}

Sign Language Phonetic Annotation (SLPA) is currently the most promising means of describing handshapes in full phonetic detail and in a machine-readable and searchable manner. In this paper, we proposed a number of changes that will help to reduce the complexity of the system, the unnecessary exhaustiveness of detail that impedes forming useful linguistic generalizations and encourages human error, as well as make it more user-friendly. The general proposals that we have made can be in principle implemented by any interested user, even if s/he uses the original system in the most straightforward way 
of manual coding. Additional proposals discussed above will hopefully be implemented in Phonological CorpusTools, a free software that will be readily available to both sign language and gesture researchers as well as Deaf communities. Even though these proposals are PCT-specific, they can be implemented in other software as well. By implementing such modifications, SLPA can become the much-needed tool for sign-language cross-linguistic annotations, and can facilitate research in sign phonetics and phonology. We hope to encourage more researchers to use SLPA, as well as to come up with other modifications that can make SLPA even more powerful.

\section{References}

Ann, Jean. (2000). Frequency of Occurrence and Ease of Articulation of Sign Language Handshapes. Gallaudet University Press.

Battison, Robbin. (1978). Lexical Borrowing in American Sign Language. Silver Spring: Linstok Press.

Baus, Christina, Eva Gutiérrez-Sigut, Josep Quer, \& Manuel Carreiras. (2008). Lexical access in Catalan signed language (LSC) production. Cognition, 108(3), 856-865.

Brentari, Diane. (2006). Effects of language modality on word segmentation: An experimental study of phonological factors in a sign language. Papers in laboratory phonology, 8, 155-164.

Carreiras, Manuel, Eva Gutiérrez-Sigut, Silvia Baquero, \& David Corina. "Lexical processing in Spanish sign language (LSE)." Journal of Memory and Language 58, no. 1 (2008): 100-122.

Corina, David. (2000). "Some observations regarding paraphasia in American Sign Language." The signs of language revisited: An anthology to honor Ursula Bellugi and Edward Klima, pp. 493-507.

Crasborn, Onno. (2012) "Phonetics." In: Pfau, Roland, Markus Steinbach \& Bencie Woll (eds.) Sign language. An international handbook. (HSK - Handbooks of linguistics and communication science), pp. 4-20. Berlin: Mouton de Gruyter.

Eccarius, Petra, \& Diane Brentari. (2008). Handshape coding made easier A theoretically based notation for phonological transcription. Sign Language \& Linguistics, 11(1), 69-101.

Eccarius, Petra, Rebecca Bour, \& Robert A. Scheidt. (2012). Dataglove measurement of joint angles in sign language handshapes. Sign language and linguistics, 15(1), 39.

Frishberg, Nancy, Nini Hoiting, \& Dan I. Slobin. (2012). Transcription. In R. Pfau, M. Steinbach, and B. Woll (Eds.) Sign Language. An international handbook, 1045-1075.

Hall, Kathleen C., Blake Allen, Michael Fry, Scott Mackie, \& Michael McAuliffe. (2015). Phonological CorpusTools, Version 1.0.1. [Computer program]. Available from http://phonologicalcorpustools.github.io/CorpusTools/.

Hochgesang, Julie A. (2014) Using Design Principles to Consider Representation of the Hand in Some Notation Systems. Sign Language Studies, 14(4), 488-542.

Johnson, Robert E., \& Scott K. Liddell. (2010). Toward a phonetic representation of signs: Sequentiality and contrast. Sign Language Studies, 11(2), 241-274.

Johnson, Robert E., \& Scott K. Liddell. (2011a). A segmental framework for representing signs phonetically. Sign Language Studies, 11(3), 408-463.

Johnson, Robert E., \& Scott K. Liddell. (2011b). Toward a phonetic representation of hand configuration: the fingers. Sign Language Studies, 12(1), 5-45.

Johnson, Robert E., \& Scott K. Liddell. (2012). Toward a Phonetic Representation of Hand Configuration: The Thumb. Sign Language Studies, 12(2), 316-333.

Johnston, Trevor. (2008). Corpus linguistics and signed languages: no lemmata, no corpus. In 3rd Workshop on the Representation and Processing of Sign Languages.

Marentette, Paula, \& Rachel Mayberry. (2000). "Principles for an emerging phonological system: A case study of language acquisition by eye." Language acquisition by eye. Mahwah, NJ: Lawrence Erlbaum.

Meier, Richard P. (2000). "Shared motoric factors in the acquisition of sign and speech." The signs of language revisited: An anthology to honor Ursula Bellugi and Edward Klima, pp. 333-356.

Prillwitz, Siegmund, Regina Leven, Heiko Zienert, Thomas Hanke, \& Jan Henning. (1989). Hamburg notation system for sign languages. An introductory guide, HamNoSys version 2.0. Signum, Seedorf, Germany.

Sandler, Wendy, \& Diane Lillo-Martin. (2006). Sign language and linguistic universals. Cambridge University Press.

Siple, Patricia. (1978). Visual constraints for sign language communication. Sign Language Studies, 19(1), 95-110.

Stokoe, William C. (1960). Sign language structure: An outline of the visual communication systems of the American deaf. Studies in Linguistics: Occasional Papers 8. Buffalo, NY: Department of Anthropology and Linguistics, University of Buffalo.

Tyrone, Martha E. (2002). Overview of capture techniques for studying sign language phonetics. In Gesture and Sign Language in Human-Computer Interaction (pp. 101-104). Springer Berlin Heidelberg. 